\title{
Neutron Scattering in Normal and Deuterated Polyethylene*
}

\author{
J. E. Lynch, Jr., $†$ G. C. Summerfield, L. A. Fetdkamp, † and J. S. King \\ Department of Nuclear Engineering, The University of Michigan, Ann Arbor, Michigan
}

(Received 5 September 1967)

\begin{abstract}
The one- and two-phonon amplitude-weighted directional frequency functions are calculated for normal and deuterated crystalline polyethylene. These results are compared to previously measured frequency spectra for stretch-oriented normal polyethylene and to new measurements on deuterated polyethylene. In addition, the Debye-Waller factors are calculated for oriented polyethylene and compared with elasticscattering data.
\end{abstract}

\section{INTRODUCTION}

Previous efforts ${ }^{1}$ to interpret the results of neutron scattering on oriented polyethylene have shown that under certain approximations the cross section should be proportional to a directional frequency distribution function $G_{j}^{\alpha \beta}(\epsilon)$, which in turn depends on the density and polarization vectors for the normal mode vibrations. Recently, the normal-mode frequency solutions have been reported for crystalline polyethylene. ${ }^{2,3}$ The present paper reports calculations of the frequencies and polarization vectors necessary for the construction of the directional frequency functions over the complete acoustical and optical frequency range. These functions are used to calculate zero-, one-, and two-phonon terms in the usual neutron cross-section expansion and provide a direct comparison with the frequency functions and Debye-Waller factors obtained from scattering measurements. The frequency functions are compared with earlier data on stretch-oriented polyethylene and with data recently obtained for deuterated polyethylene, $\left(\mathrm{CD}_{2}\right)_{n}$. Measured Debye-Waller factors are reported and compared with calculations for normal polyethylene only.

Brief justification of the several approximations basic to the calculations is made in the following theoretical section. At the outset, however, it must be emphasized that the calculated cross sections for $\left(\mathrm{CD}_{2}\right)_{n}$ are made using the "incoherent approximation" although this is a predominantly coherent scatterer. The close similarity between the normal and deuterated spectra gives some support for such a treatment. Of course, the approximation is strictly valid only when $\kappa$ is much larger than the spacing between reciprocal lattice points-a condition not well satisfied in the present experiments.

\footnotetext{
* Work supported in part by the National Science Foundation under Grant Numbers GK 1709 and GK 201.

$\uparrow$ USAEC Predoctoral Fellow.

$¥$ NSF Predoctoral Fellow.

${ }^{\ddagger}$ G. C. Summerfield, J. Chem. Phys. 43, 1079 (1965).

$2 \mathrm{M}$. Tasumi and T. Shimanouchi, J. Chem. Phys. 43, 1245 (1965).

${ }^{3}$ M. Tasumi and S. Krimm, J. Chem. Phys. 46, 755 (1967).
}

\section{THEORY}

Following the notation of Ref. 4 we can write the cross section as

$$
\begin{aligned}
\frac{d^{2} \sigma}{d \Omega d \epsilon}=\frac{N \sigma_{0} k_{f}}{\pi k_{i}}\langle\exp [ & -Z(0)] \int_{-\infty}^{\infty} \frac{d t}{2 \pi \hbar} \\
& \times \exp (-i \epsilon t / \hbar) \exp [Z(t)]\rangle,
\end{aligned}
$$

where the symbol \langle\rangle indicates the usual thermal average plus an appropriate average over molecular orientation. $Z(t)$ is given by

$$
Z(t)=\sum_{\alpha \beta} \frac{\hbar^{2} \kappa_{\alpha} \kappa_{\beta}}{2 N m} \int_{-\infty}^{\infty} d \epsilon^{\prime} \frac{G^{\alpha \beta}\left(\epsilon^{\prime}\right)}{\epsilon^{\prime}} \frac{\exp \left(i \epsilon^{\prime} t / \hbar\right)}{1-\exp \left(-\beta \epsilon^{\prime}\right)}
$$

and

$$
\begin{aligned}
& G^{\alpha \beta}(\boldsymbol{\epsilon})=\sum_{j \mathrm{q}} \gamma_{j}^{\alpha *}(\mathbf{q}) \gamma_{j}^{\beta}(\mathbf{q}) \delta\left(\epsilon \hbar \omega_{j}(\mathbf{q})\right) \\
& G^{\alpha \beta}(\boldsymbol{\epsilon})=G^{\alpha \beta}(-\boldsymbol{\epsilon}) .
\end{aligned}
$$

For a comparison between theory and experiment, it is convenient to introduce a function related to the inelastic cross section:

$$
\begin{aligned}
G_{\kappa}(\epsilon)=\left(2 m \pi k_{i} / \sigma_{0} \hbar^{2} \kappa^{2} k_{f}\right) \epsilon[1- & \exp (-\beta \epsilon)] \\
& \times\left(d^{2} \sigma / d \Omega d \epsilon\right) \text { inelastic. }
\end{aligned}
$$

This can be written in terms of $Z(t)$ as

$$
\begin{gathered}
G_{\kappa}(\epsilon)=\left\langle\frac{2 m N}{\hbar^{2} \kappa^{2}} \epsilon[1-\exp (-\beta \epsilon)] \exp [-Z(0)] \int_{-\infty}^{\infty} \frac{d t}{2 \pi \hbar}\right. \\
\left.\times \exp \left(-\frac{i \epsilon t}{\hbar}\right)\{\exp [Z(t)]-1\}\right\rangle
\end{gathered}
$$

For stretched polyethylene, only the chain axis is oriented, and that is along the stretch direction. Thus for a stretched target, we need only consider two functions: One for $\boldsymbol{x}$ directed along the stretch direction,

${ }^{4}$ W. Myers, G. C. Summerfield, and J. S. King, J. Chem. Phys. 44, $184(1966), \sigma_{0}$ is the free-atom cross section for hydrogen and $1 / 4$ of the bound-atom cross section for other nuclei. 
Fig. 1. Transverse frequency function $G_{T}{ }^{I}(\epsilon)$ for a hydrogen atom in normal polyethylene. $\left(G_{T} I=\right.$ 0.047 at $0.002 \mathrm{eV}$.)

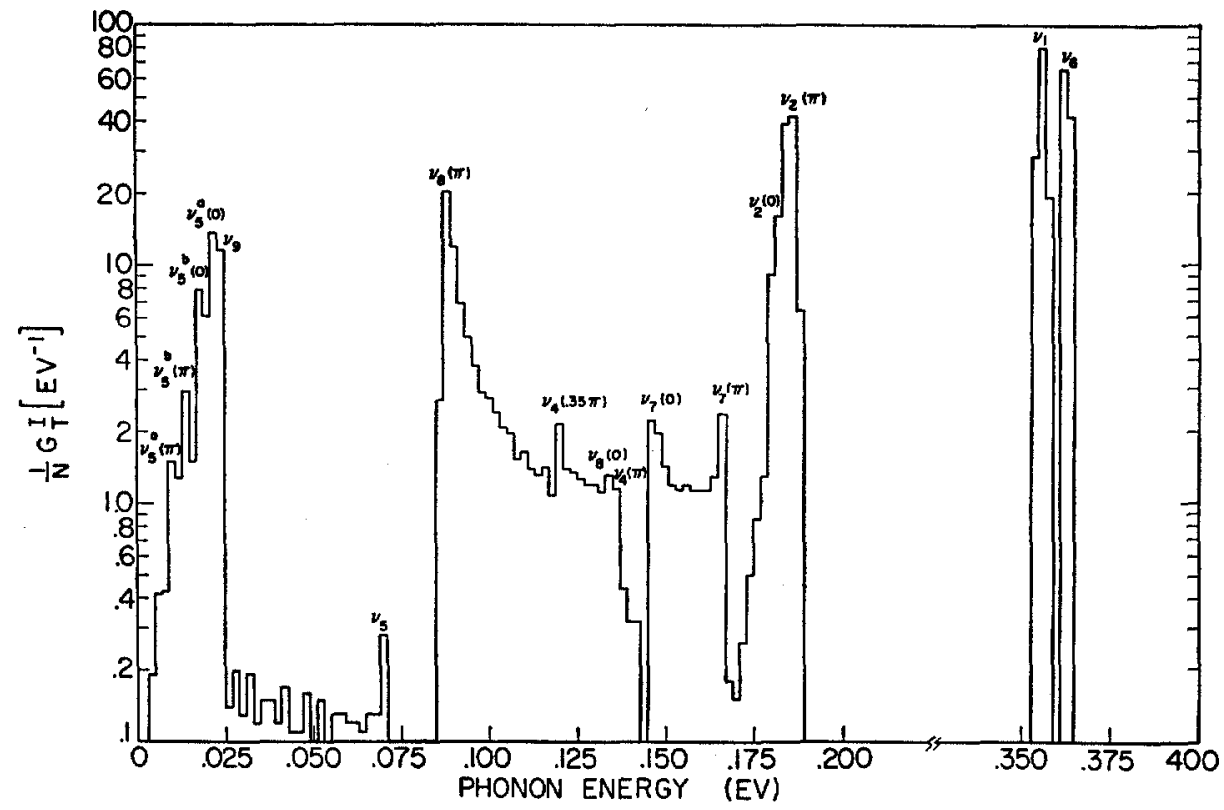

$G_{L}(\epsilon)$, and one for $\boldsymbol{x}$ directed perpendicular to the stretch direction, $G_{T}(\epsilon)$. For an unstretched target, even the chain axes are unoriented and we obtain a function independent of the directions of $\boldsymbol{x}, G(\epsilon)$.

If we take the $\alpha=1$ axis along the chain axis, then we can define three functions

$$
\begin{aligned}
G_{L}{ }^{I}(\epsilon) & =G^{11}(\epsilon), \\
G_{T} I(\epsilon) & =\frac{1}{2}\left\{G^{22}(\epsilon)+G^{33}(\epsilon)\right\}, \\
G^{I}(\epsilon) & =\frac{1}{3}\left\{G^{11}(\epsilon)+G^{22}(\epsilon)+G^{23}(\epsilon)\right\} .
\end{aligned}
$$

The functions $G^{22}(\epsilon)$ and $G^{33}(\epsilon)$ are almost identical, so to a good approximation we can write the transverse cross section in terms of $G_{T} T(\epsilon)$. Of course, the cross section for the longitudinal orientations of $\boldsymbol{x}$ can be written in terms of $G_{L}{ }^{I}(\epsilon)$ without any approximations.

For the longitudinal cross section, the factor $\exp [-Z(0)]$ can be written

where

$$
\exp [-Z(0)]=\exp \left(-2 W_{L}\right)
$$

$$
2 W_{L}=\int_{0}^{\infty} \frac{\hbar^{2} \kappa^{2}}{2 m N} \frac{\operatorname{coth}(\beta \epsilon / 2)}{\epsilon} G_{L}{ }^{I}(\epsilon) d \epsilon
$$

FIG. 2. Longitudinal frequency function $G_{L}^{I}(\epsilon)$ for a hydrogen atom in normal polyethylene. $\left(G_{L}{ }^{I}=\right.$ 0.033 at $0.002 \mathrm{eV}$.)

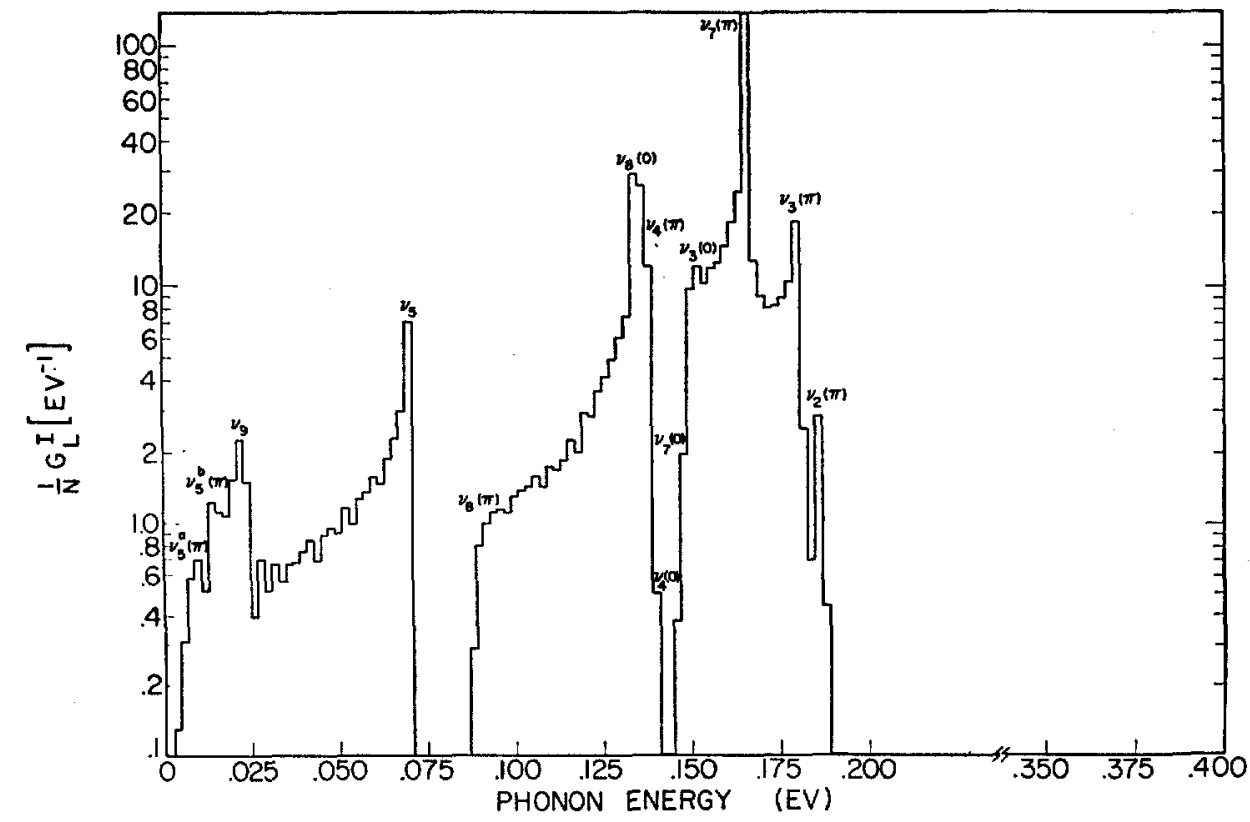




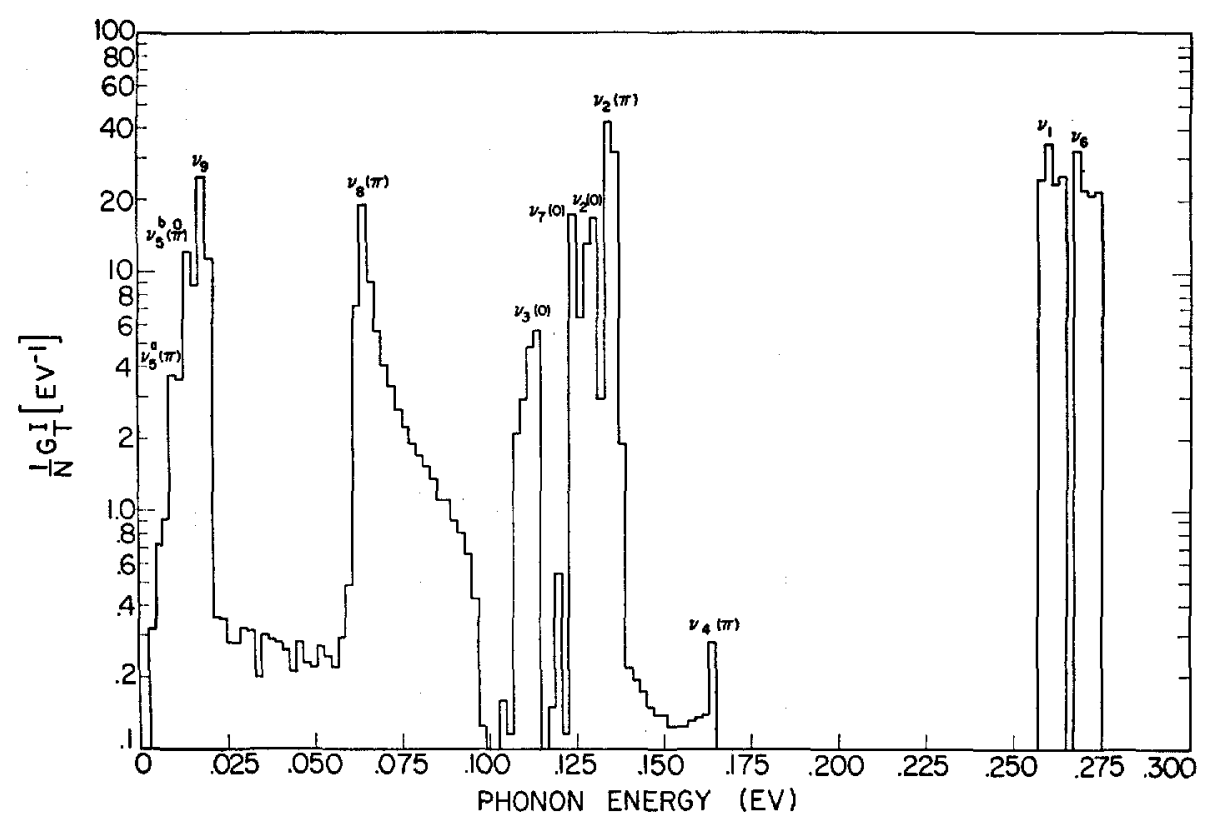

FIG. 3. Transverse frequency function $G_{T}^{I}(\epsilon)$ for a deuterium atom in deuterated polyethylene. $\left(G_{T} I\right.$ $=0.08$ at $0.002 \mathrm{eV}$.)
For the transverse cross section, this factor is well approximated by

$$
\exp [-Z(0)] \sim \exp \left(-2 W_{T}\right),
$$

where

$$
2 W_{T}=\int_{0}^{\infty} \frac{\hbar^{2} \kappa^{2}}{2 m N} \frac{\operatorname{coth}(\beta \epsilon / 2)}{\epsilon} G_{T} I(\epsilon) d \epsilon .
$$

\section{CALCULATIONS}

The stretched targets are assumed to consist of microcrystals with chain axes nearly parallel to the stretch direction and randomly oriented in directions perpendicular to the chain axes. This model is in agreement with both $x$-ray and neutron-diffraction observations. The directional $G$ functions are constructed from the eigenfrequency and eigenvector solutions to the dynamical matrix equation for the hydrogen motions in one such microcrystal. The Cartesian coordinate $G F$-matrix method described in Ref. 5 for optically active lattice vibrations may be adapted so that the matrix equation in Cartesian symmetry coordinates is

$$
\mathrm{C}^{8} \boldsymbol{\Gamma}_{c}^{8}=\boldsymbol{\Gamma}_{c}^{s} \boldsymbol{\Lambda},
$$

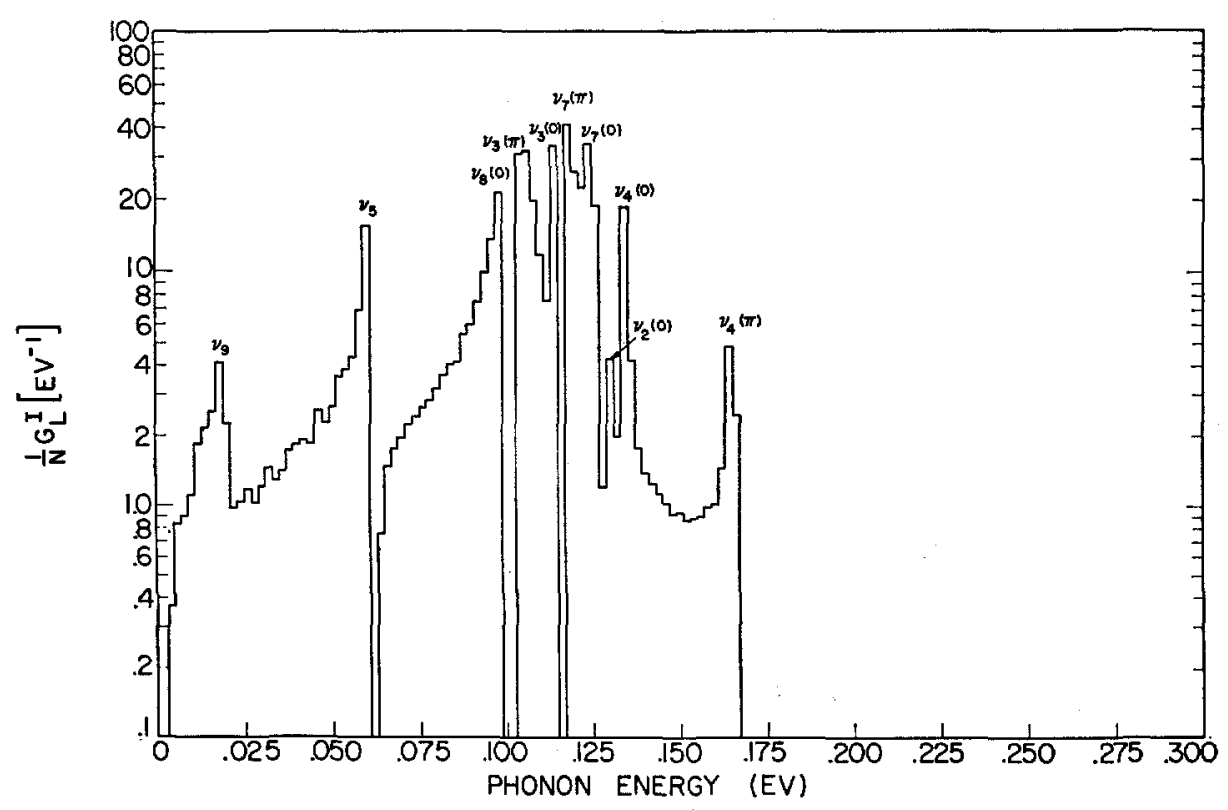

FIG. 4. Longitudinal frequency function $G_{L}{ }^{I}(\epsilon)$ for a deuterium atom in deuterated polyethylene. $\left(G_{L} I=\right.$ 0.09 at $0.002 \mathrm{eV}$.)

5. Shimanouchi, M. Tsuboi, and T. Miyazawa, J. Chem. Phys. 35, 1597 (1961). 


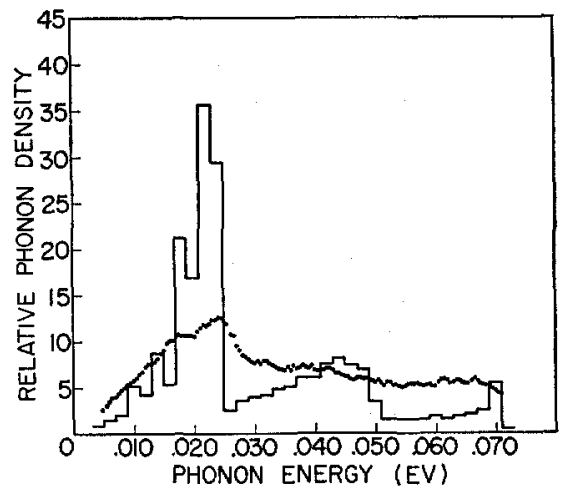

FIG. 5. Frequency function $G(\epsilon)$ for unoriented normal polyethylene. [Experimental points from Ref. 4 for $E_{f}=0.030 \mathrm{eV}$, $\left.T=100^{\circ} \mathrm{K}, \phi=90^{\circ} ; G(\epsilon)=\frac{1}{8} G_{L}(\epsilon)+\frac{2}{3} G_{T}(\epsilon).\right]$

where

and

$$
\begin{aligned}
\mathbf{C}^{\delta} & =\mathrm{U}\left(\delta_{c}\right) \mathrm{C}( \pm) \mathrm{U}\left(\delta_{c}\right)^{\dagger}, \\
\mathrm{C}( \pm) & =\mathbf{M}^{-1 / 2} \mathrm{~F}_{c}{ }^{s} \mathbf{M}^{-1 / 2},
\end{aligned}
$$

$$
\mathrm{F}_{c}^{8}=\mathrm{B}^{\varepsilon+} \mathrm{FB}^{s}+\mathrm{B}^{\prime s+} \mathrm{F}^{\prime} \mathbf{B}^{\prime s} \text {. }
$$

Unit-cell dimensions appropriate to liquid-nitrogen temperatures are used, ${ }^{\circ}$ with a molecular setting angle of $\theta=48^{\circ}$. True, nontetrahedral coordinates are used for the molecular chains. ${ }^{7}$ The $\mathbf{B}$ and $\mathbf{B}^{\prime}$ matrix elements are calculated for the intramolecular internal coordinates $^{8}$ and intermolecular internal coordinates, ${ }^{2}$ using a computer method similar to that employed by Overend and Scherer. 9 The $B^{s}$ and $B^{\prime s}$ matrices in Cartesian symmetry coordinates are then obtained by simply altering the signs of elements in the $a$ and $b$ Cartesian coordinate columns as required.

The symmetry transformation is then performed on the internal-coordinate intramolecular-force-constant

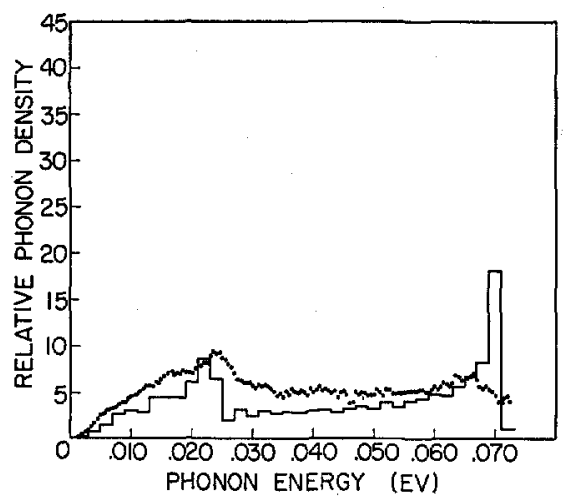

FIG. 6. Longitudinal frequency function $G_{L}(\epsilon)$ for oriented normal polyethylene. [Experimental points from Ref. 4; $E_{f}=$ $0.030 \mathrm{eV}, T=100^{\circ} \mathrm{K}, \phi=90^{\circ}$.]

'P. R. Swan, J. Polymer Sci. 56, 403 (1962).

7 P. W. Teare, Acta Cryst. 12, 294 (1959).

${ }^{8} \mathrm{M}$. Tasumi, T. Shimanouchi, and T. Miyazawa, J. Mol. Spectry. 9, 261 (1962).

J. Overend and J. R. Scherer, J. Chem. Phys. 32, 1289 (1960).

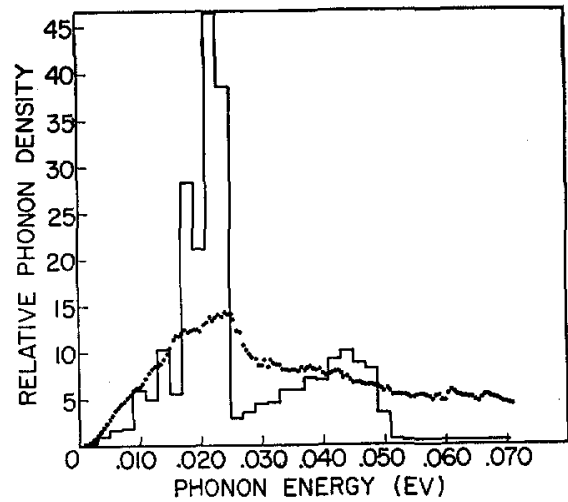

FIG. 7. Transverse frequency function $G_{T}(\epsilon)$ for oriented normal polyethylene. [Experimental points from Ref. $4 ; E_{f}=0.030 \mathrm{eV}$, $\left.T=100^{\circ} \mathrm{K}, \phi=90^{\circ}.\right]$

matrix $(F)$ of Schachtschneider and Snyder ${ }^{10}$ which is not altered except for the diagonal internal-rotation force constant. This force constant is changed from 0.008 to $0.05 \mathrm{mdyn} \cdot \AA / \mathrm{rad}^{2}$ for the coordinate $\Delta t=$ $\sum\left(\Delta t_{i j / 9}\right) .^{11}$ The $\mathbf{B}^{\prime s}$ matrices are also applied to the internal-coordinate interaction-force-constant matrix ${ }^{3}$ $\left(F^{\prime}\right)$ so that finally Eq. (15) is obtained. The $C( \pm)$ matrix is then formed as in Eq. (14).

Solutions to the block-diagonal matrix equation [Eq. (13)] are obtained at $200 \delta_{c}$ phase values, when $0 \leq \delta_{c} \leq \pi$, for both normal and deuterated polyethylene. Directional frequency functions are calculated as histograms in $0.002-\mathrm{eV}$ increments. The functions $G_{T} I(\epsilon)$ and $G_{L} I(\epsilon)$ are displayed in Figs. 1 and 2 for normal polyethylene at liquid-nitrogen temperature. The frequency spectra from $\epsilon=0$ to $0.006 \mathrm{eV}$ are given a quadratic frequency dependence, in accord with specificheat requirements ${ }^{12}$ and in order to guarantee a finite Debye-Waller coefficient and nonzero cross section.

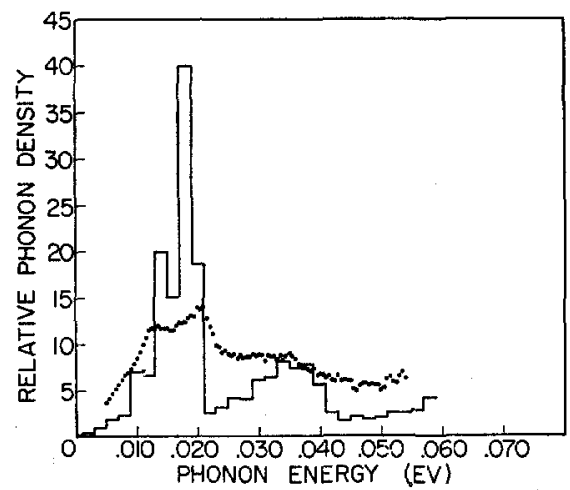

FIG. 8. Frequency function $G(\epsilon)$ for unoriented deuterated polyethylene. [Experimental points are the average of values for $E_{f}=0.028 \mathrm{eV}$ and $E_{f}=0.031 \mathrm{eV}$ obtained at $78^{\circ} \mathrm{K}, \phi=89^{\circ}$, $\left.G_{\text {otio }}(\epsilon)=\frac{1}{3} G_{L}(\epsilon)+\frac{2}{3} G_{T}(\epsilon).\right]$

${ }^{10} \mathrm{~J}$. H. Schachtschneider and R. G. Snyder, Spectrochim. Acta 19, 117 (1963).

11 T. Miyazawa and K. Fukushima, J. Mol. Spectry. 15, 308 (1965).

12 B. Wunderlich, J. Chem. Phys. 37, 1207 (1962). 


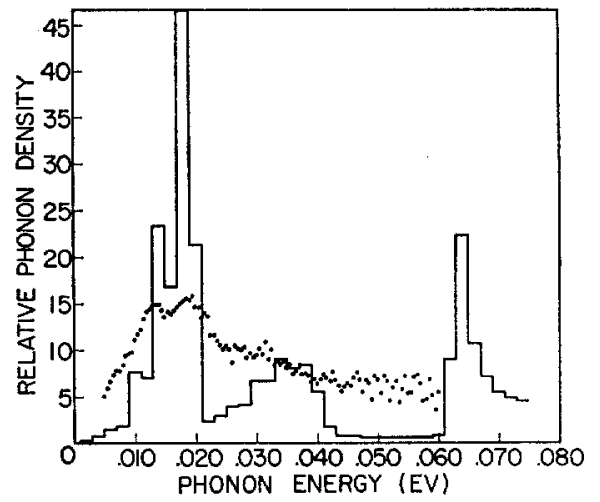

FIG. 9. Transverse-frequency function $G_{T}(\epsilon)$ for deuterated polyethylene. (Experimental points obtained for $E_{f}=0.028 \mathrm{eV}$, $T=78^{\circ} \mathrm{K}, \phi=89^{\circ}$.)

The only change made in solving the dynamical matrix for $\left(\mathrm{CD}_{2}\right)_{n}$ was to replace the hydrogen mass with the deuterium mass. The functions $G_{T}^{I}(\epsilon)$ and $G_{L}{ }^{I}(\epsilon)$ are displayed in Figs. 3 and 4 for deuterated polyethylene.

The functions $G_{L}(\epsilon), G_{T}(\epsilon)$, and $G(\epsilon)$ are computed from Eq. (5) using one- and two-phonon contributions $\left[e^{Z}(t)=1+Z(t)+\frac{1}{2} Z^{2}(t)\right]$. The results are shown in Figs. 5-9. However, note that the measurements of $G(\epsilon)$ are compared to calculated values of $\frac{1}{3} G_{L}(\epsilon)+$ $\frac{2}{3} G_{T}(\epsilon)$ in Figs. 5 and 8.

\section{EXPERIMENTAI DATA}

The inelastic-scattering measurements on lowtemperature oriented polyethylene have been published previously ${ }^{4}$; the major features are in agreement with the room-temperature results of Trevino. ${ }^{18}$ The present work includes: (a) a re-examination of the elasticscattering part of the data, extended to include an angular range from $55^{\circ}$ to $90^{\circ}$, and (b) inelastic scattering from fully deuterated polyethylene, $\left(\mathrm{CD}_{2}\right)_{n}$. The University of Michigan triple-axis crystal spectrometer was used for these measurements.

The variation in elastic-scattering cross sections with momentum transfer for both the Iongitudinal and transverse normal polyethylene was measured in order to determine the Debye-Waller coefficients $a_{L}=2 W_{L} / \kappa^{2}$ and $a_{T}=2 W_{T} / \kappa^{2}$. An analyzer energy of $0.0548 \mathrm{eV}$ was selected to avoid significant second-order contamination. The target was oriented within the liquid-nitrogen cryostat with the $c$ axis either parallel or perpendicular to the direction of $\boldsymbol{x}$. Small deviations from precise orientation were shown to affect the scattered (incoherent) intensity by less than $1 \%$.

The elastic cross section is taken to be proportional to the area under the elastic peak after subtraction of background and an inelastic component, estimated by a smooth curve extension of the inelastic wings. A correction for target thickness and beamwidth variation was also applied. The corrections introduce an unS. F. Trevino, J. Chem. Phys. 45, 757 (1966). certainty in the inferred coefficients of about $10 \%$. A plot of the logarithm of the intensity versus $\kappa^{2}$ for each orientation is shown in Fig. 10. The scatter in the data points is clearly greater than expected from counting statistics and is attributed to the varying importance of the small coherent cross section in polyethylene. The anomalously high points in the longitudinal data, at $\kappa^{2}=29.04$ and $27.19 \mathrm{do}$, in fact, correlate roughly with two structure-factor peak positions for the crystal unit cell. Further, the high density of unresolved points in reciprocal space at the larger angles makes this region suspect. As a result, the straight lines shown in Fig. 10 are based on two criteria: (a) points believed to be most seriously affected by coherent scattering are given low weight, and (b) the intercepts for both orientations should be equal at $\kappa^{2}=0$ since the two incoherent cross sections approach the same value there.

Deuterated targets were prepared from $25 \mathrm{~g}$ of granules (Volk Chemical Corporation, $98 \%$ deuterated) by pressing at approximately $160^{\circ} \mathrm{K}$ for $5-10 \mathrm{~min}$ at 1000 pounds per square inch. A polycrystalline target 3 in. diam $\times 0.144$ in. thick (calculated $0.05-\mathrm{eV}$ transmission, $90 \%$ ) was used for the unoriented measurements. Oriented targets were prepared by stretching $500 \%$ under infrared heat $\left(T \leq 100^{\circ} \mathrm{C}\right)$. Greater stretching resulted in rupture under tension. An oriented target 3 in. $\times 5$ in. $\times 0.086$ in. was used. Such stretching, unfortunately, produces a rather wide distribution of crystallite $c$-axis orientations; neutron diffraction from the (002) reflection showed a FWHM of $8^{\circ}$. X-ray patterns for both the normal and deuterated stretched samples look similar and qualitatively confirm the neutron width.

All measurements were made with a fixed scattering angle of $89^{\circ}$ and a target temperature of $78^{\circ} \mathrm{K}$. The polycrystalline data comprise an average of two separate measurements at final energies of 0.028 and 0.031 $\mathrm{eV}$; the transverse data were all taken with $E_{f}=0.028$ $\mathrm{eV}$. The energy resolution of the spectrometer at $0.02-\mathrm{eV}$ energy transfer is $0.0013 \mathrm{eV}(6.5 \%)$.

The measured frequency functions, obtained from the incoherent approximation, are shown in Fig. 8 for the unoriented case and in Fig. 9 for the transverse-

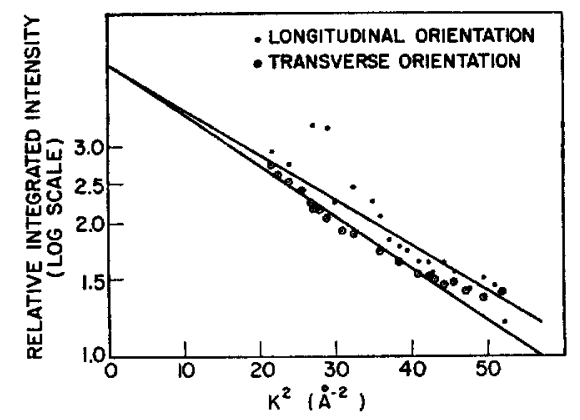

Fig. 10. Relative elastic-scattering cross section vs $k^{2}$ for longitudinal and transverse orientations of the stretched normal polyethylene target. 
oriented case. Figure 11 compares the unoriented frequency functions for normal and deuterated polyethylene.

Thus far, no single-phonon events have been observed for the deuterated target, although scans have been made with $\times$ along the $c$ axis. This is believed due partly to "smearing" from the wide mosaic and partly to the interference from elastic scattering which "leaks" into the detector due to incoherent scattering in the analyzer crystals.

\section{DISCUSSION}

The comparison of measured and calculated frequency functions, Figs. 5-9, can be said to show qualitative agreement only. As yet the spectrometer resolution has not been removed from the data nor folded into the calculated curves (the latter already have substantial width from the computer code mesh); this resolution effect clearly dampens the calculated structure, particularly at the $\left(\nu_{5}\right)$ limit near $0.065 \mathrm{eV}$. However, the differences between experiment and theory are too large to be attributed solely to resolution. Despite the damping, however, several main features show reasonable agreement in all the normal polyethylene comparisons. From the data we note: (a) the peak near $0.024 \mathrm{eV}$, (b) the shoulder at about $0.017 \mathrm{eV}$, (c) the broad maximum near $0.040 \mathrm{eV}$, and (d) the very broad peak near $0.065 \mathrm{eV}$ in Fig. 6. These events correlate well with known features in the calculations. Relative to the above data these are, respectively: (a) the $\left(\nu_{9}\right)$ maximum frequency cutoff [the $\left(\nu_{6}\right)$ intercept $\nu_{b}{ }^{a}(0)$ occurs very near this cutoff and would not be resolved], (b) one of the intercepts at 0 or $\pi$ phase for the crystalline mode $\nu_{5}^{b}$, most probably $\nu_{5}^{b}(0)$ from the relative response of longitudinal and transverse magnitudes, (c) the broad two-phonon maximum near $0.044 \mathrm{eV}$, which correlates only approximately with the experimental choice of $0.040 \mathrm{eV}$, (d) the frequency limits for both crystalline branches of $\nu_{5}$, which occur indistinguishably close together at $0.068 \mathrm{eV}$ in the longitudinal data. With regard to (d),

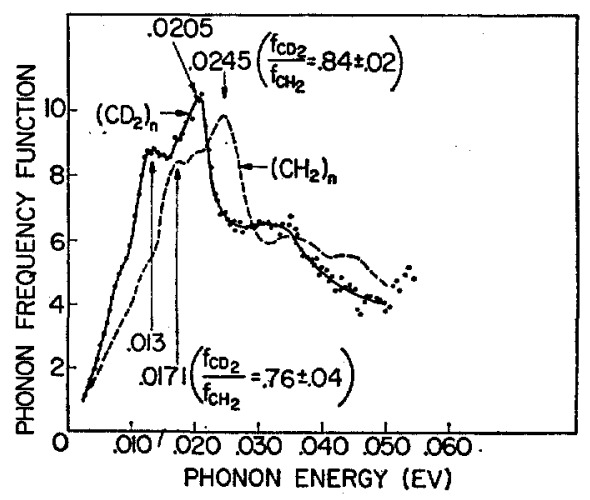

FiG. 11. Frequency shifts for deuterated polyethylene (unoriented $\mathrm{CD}_{2}$ samples at $78^{\circ} \mathrm{K}, \mathrm{CH}_{2}$ at $90^{\circ} \mathrm{K}$; scattered angle $\phi=90^{\circ}, E_{f}=0.030 \mathrm{eV}$ ).
Table I. Measured and calculated Debye-Waller coefficients fơr normal polyethylene.

\begin{tabular}{lllll}
\hline & \multicolumn{1}{c}{$a_{L}$} & $a_{T}$ & & \\
& $=2 W_{L} / \kappa^{2}$ & $=2 W_{T} / \kappa^{2}$ & $a_{T}-a_{L}$ & $e^{-2 W_{L}} / e^{-2 W T}$ \\
\hline Measured & $0.024 \AA^{2}$ & $0.027 \AA^{2}$ & $0.0028 \AA^{2}$ & 1.09 \\
Calculated & 0.0205 & 0.0253 & 0.0048 & 1.15 \\
\hline \hline
\end{tabular}

no attempt has been made to adjust the internalcoordinate intramolecular force constants ${ }^{10}$ to effect a better match with the experimental choice of $0.065 \mathrm{eV}$. In addition to these events, the results give some evidence to confirm the calculated discontinuities near $0.013 \mathrm{eV}$, associated with $\nu_{5}^{b}(\pi)$, and near $0.009 \mathrm{eV}$, associated with $\nu_{5}^{a}(\pi)$. However, the data are badly resolved and firm comparisons cannot yet be made.

A similar comparison (Fig. 8) for the deuterated targets can be made. The structure has somewhat greater clarity and provides strong confirmation for the appearance of the $\left(\nu_{9}\right)$ limit, and the intercepts for $\nu_{5}^{b}(0$ or $\pi)$, and $\nu_{5}{ }^{a}(\pi)$. The two-phonon maximum again appears and again is somewhat below calculation. The downward shift in all frequencies relative to normal polyethylene is best seen in Fig. 11. The frequency ratios, particularly, should be accurate. These show values of $0.84 \pm 0.02$ for the $\nu_{9}$ cutoff, and $0.76 \pm 0.04$ for the $\nu_{5}^{b}$ intercepts. These ratios are in excellent agreement with those calculated by Tasumi and $\mathrm{Krimm}^{3}$ for $\left(\nu_{9}\right)$ and $\nu_{5}^{b}(0)$. They, therefore, represent to some degree a low-frequency experimental confirmation of the Tasumi-Krimm model.

A comparison of the Debye-Waller coefficients and Debye-Waller ratios at approximately $93^{\circ} \mathrm{K}$ is given in Table I for normal polyethylene. In view of the coherent interference noted above, the agreement is within experimental limits of accuracy. It should be noted that at $93^{\circ} \mathrm{K}$, major portions of the coefficients are contributed by branches with frequencies above $600 \mathrm{~cm}^{-1} ; 60 \%$ of the value for $2 W_{L}$ and $40 \%$ of that for $2 W_{T}$ comes from this high-frequency region.

In conclusion, it may be said that the agreement between the calculation and experiment (Figs. 5-9) is as good as should be expected at this stage of refinement. Better resolution in the experimental data is clearly needed. At the same time the theoretical analysis is based on an idealized model for polyethylene, i.e., a perfect, harmonic crystal. The forces, particularly the torsional forces, are not harmonic and the carbon chains exhibit folding. Some work has been done on the latter effect, ${ }^{14}$ but little has been done on anharmonicities and these should be particularly significant for the torsional modes below $0.025 \mathrm{eV}$. Finally, the restriction that the phonon-wave vector lies along the chain axis must be evaluated; removal of this restriction should tend to lower the cutoff frequencies for $\nu_{9}$ and $\nu_{5}$.

\footnotetext{
${ }^{14}$ G. Jannink and G. C. Summerfield, J. Appl. Phys. 37, 3953 (1966).
} 